\title{
Influence of stochastic domain growth on pattern nucleation for diffusive systems with internal noise
}

\author{
Thomas E. Woolley, ${ }^{1, *}$ Ruth E. Baker, ${ }^{1}$ Eamonn A. Gaffney, ${ }^{1}$ and Philip K. Maini ${ }^{1,2}$ \\ ${ }^{1}$ Centre for Mathematical Biology, Mathematical Institute, University of Oxford, 24-29 St Giles', Oxford OXI 3LB, United Kingdom \\ ${ }^{2}$ Oxford Centre for Integrative Systems Biology, Department of Biochemistry, University of Oxford, \\ South Parks Road OX1 3QU, United Kingdom
}

(Received 6 April 2011; revised manuscript received 27 May 2011; published 3 October 2011)

\begin{abstract}
Numerous mathematical models exploring the emergence of complexity within developmental biology incorporate diffusion as the dominant mechanism of transport. However, self-organizing paradigms can exhibit the biologically undesirable property of extensive sensitivity, as illustrated by the behavior of the French-flag model in response to intrinsic noise and Turing's model when subjected to fluctuations in initial conditions. Domain growth is known to be a stabilizing factor for the latter, though the interaction of intrinsic noise and domain growth is underexplored, even in the simplest of biophysical settings. Previously, we developed analytical Fourier methods and a description of domain growth that allowed us to characterize the effects of deterministic domain growth on stochastically diffusing systems. In this paper we extend our analysis to encompass stochastically growing domains. This form of growth can be used only to link the meso- and macroscopic domains as the "box-splitting" form of growth on the microscopic scale has an ill-defined thermodynamic limit. The extension is achieved by allowing the simulated particles to undergo random walks on a discretized domain, while stochastically controlling the length of each discretized compartment. Due to the dependence of diffusion on the domain discretization, we find that the description of diffusion cannot be uniquely derived. We apply these analytical methods to two justified descriptions, where it is shown that, under certain conditions, diffusion is able to support a consistent inhomogeneous state that is far removed from the deterministic equilibrium, without additional kinetics. Finally, a logistically growing domain is considered. Not only does this show that we can deal with nonmonotonic descriptions of stochastic growth, but it is also seen that diffusion on a stationary domain produces different effects to diffusion on a domain that is stationary "on average."
\end{abstract}

DOI: 10.1103/PhysRevE.84.041905 PACS number(s): 87.15.Vv, 82.20.Uv, 87.18.Tt, 02.50.Fz

\section{INTRODUCTION}

Understanding the emergence of structure on scales much greater than that of an individual cell is a core feature of developmental biology and requires the orchestration of cellular behavior and differential gene expression over large length scales. Diffusible biochemicals, known as morphogens, provide a fundamental means of controlling the concomitant long-range cellular signaling [1] and have been the subject of numerous mathematical modeling explorations, based on continuum models of reaction and diffusion [2]. One critical question of all hypothesized mechanisms and their mathematical representations that is difficult to address intuitively is whether they exhibit an unreasonable sensitivity to small perturbations given the need for robustness in most developmental settings. For instance, Turing's eponymous model [3] for self-organization via two interacting morphogens yields different predictions given fluctuations in domain geometries and initial conditions [4,5], whereas domains of differential gene expression based on morphogen levels are potentially sensitive to internal noise in the French-flag model $[6,7]$.

Robustness to initial conditions in deterministic Turing patterning systems can be restored by domain growth, which instigates a sequential pattern doubling mechanism [8]. Furthermore, embryonic and organism growth have also been hypothesized to play an important role in many areas of biological self-organization, for instance, alligator tooth ini-

*woolley@maths.ox.ac.uk tiation [9,10], fish pigmentation patterning [11], and limb-bud development [12].

In contrast, the interaction of internal noise and domain growth, even in the consideration of simple morphogen diffusion, is underexplored. An initial study used a spacejump process, where particles undergo a random walk on a discretized domain, as a description of microscale diffusion. Growth was then applied through a "box-splitting" mechanism that, for uniform growth, would replace a random compartment with two new daughter compartments of the same size. This was then accompanied by a redistribution of the parent population into the daughter boxes [13]. However, such approaches do not facilitate explorations of the weak noise limit as the box-splitting nonperturbatively alters the system, as detailed in our previous study [14] where we introduce a Lagrangian mesoscale formulation of space-jump diffusive dynamics on a growing domain. The associated chemical master equation (CME) captures interactions between diffusion and deterministic domain growth, while allowing a linear, weak-noise, van Kampen expansion [15]. Through this expansion method we derived deterministic equations, which control the mean-field dynamics, and a Fokker-Planck equation that describes the stochastic effects. Due to the linearity and symmetry of these equations, we were able to apply Fourier techniques to derive analytical expressions for temporal and spatial power spectra in a study of noise-induced nucleation of spatial heterogeneity in diffusive systems accompanied by deterministic domain growth. Although we are using linear analysis to understand nonlinear complexity, it has been shown that linear dynamics 
can often give us useful insights about the full nonlinear system $[2,16,17]$.

Here we generalize the Lagrangian mesoscale formulation of space-jump processes to incorporate stochastic domain growth. This is to assess whether the nucleation of structure by the interaction of diffusion and internal noise is sensitive to this additional aspect of stochastic dynamics. Note that, due to the lack of a link between the Eulerian and Lagrangian scales, the description of density distribution must stay on the mesoscopic scale. Furthermore, since jump rates are contingent on a stochastic compartmental size for a fixed macroscale diffusion coefficient, richer dynamics are possible. For example, a description of stochastic diffusion on the mesoscopic scale, which gives the deterministic dynamics in the weak-noise limit, cannot be uniquely derived. Consequently, our aim is to construct a space-jump representation of diffusion on a stochastically growing domain, while analytically and numerically exploring fundamental features of the model formalism. Although the framework is described in the context of molecular level particles, it is in fact more general and can be applied to any system where motility can be considered as a diffusive process governed by an unbiased random walk [18-20].

We start, in Secs. II and III, by briefly recapitulating the basic stochastic framework, the spatial Fourier transform, and the spatial mapping. For an expanded discussion on these topics see Ref. [14]. Due to the description of diffusion being nonunique, we consider two potential descriptions in Sec. IV. Although these descriptions are the same in the mean field, they have different stochastic effects, as seen in Sec. V. These examples are then extended in Sec. VI by allowing stochastic growth to be nonmonotonic. We see that diffusion on a stationary domain produces different effects to diffusion on a domain that is stationary "on average." Finally, in Sec. VII, we collect these results and interpret them, with a view to understanding the effects of stochasticity on growth and diffusive systems.

\section{STOCHASTIC FRAMEWORK}

In this section we recapitulate the basic methods that will allow analytical treatment of stochastic models (see Ref. [14] for further details).

Let a system contain $K$ chemical populations, $\left\{S_{1}, \ldots, S_{K}\right\}$, with $U_{i}(t)$ representing the number of individuals of species $S_{i}$. Further, assume that these populations are undergoing $J$ reactions, $\left\{R_{1}, \ldots, R_{J}\right\}$, which are defined by their stoichiometric vectors, $\boldsymbol{v}_{j}=\left(v_{1 j}, \ldots, v_{K j}\right)$, and their propensity functions, $a_{j}(\boldsymbol{u})$, where $\boldsymbol{u}$ is a single realization of $\boldsymbol{U}=$ $\left(U_{1}(t), \ldots, U_{K}(t)\right)[21]$. Then the probability, $P(\boldsymbol{u}, t)$, of being in a state $\boldsymbol{u}$ at a time $t$ can be defined by the chemical master equation (CME):

$$
\begin{aligned}
\frac{\partial}{\partial t} P\left(\boldsymbol{u}, t \mid \boldsymbol{u}_{0}, t_{0}\right)= & \sum_{j=1}^{J}\left[P\left(\boldsymbol{u}-\boldsymbol{v}_{j}, t \mid \boldsymbol{u}_{0}, t_{0}\right) a_{j}\left(\boldsymbol{u}-\boldsymbol{v}_{j}\right)\right. \\
& \left.-P\left(\boldsymbol{u}, t \mid \boldsymbol{u}_{0}, t_{0}\right) a_{j}(\boldsymbol{u})\right] \\
\stackrel{\text { def }}{=} & \sum_{j=1}^{J}\left(R_{j}-1\right)\left[P\left(\boldsymbol{u}, t \mid \boldsymbol{u}_{0}, t_{0}\right) a_{j}(\boldsymbol{u})\right]
\end{aligned}
$$

which encapsulates the idea that the rate of change of $P(\boldsymbol{u}, t)$ over time is equal to the net probability flux into the state $\boldsymbol{u}$. Note that here we are using $R_{j}$ to denote the reaction path, as well as the reaction operator. This abuse of notation should not cause confusion.

Since, in this paper, we are specifically dealing with a single spatially distributed particle type $U$, the $K$ chemical populations arise from the discretization of the parent population into $K$ compartments. Thus, the subscript $i$ indexes the spatial location of the compartments as well as the population. In the case of multiple different particle populations, e.g., $U, V, W$, etc., each type is discretized into $K$ boxes, with $U_{i}, V_{i}, W_{i}$, etc., inhabiting the $i$ th compartment.

\section{A. Weak noise expansion}

Stochastic systems that model nonlinear spatial and temporal dynamics rarely have analytically solvable CMEs; thus, we systematically approximate the CME by expanding it in terms of a suitable system parameter. Here the expansion variable is denoted $\Omega$ and is defined as the initial mean value of the single particle type, $U_{i}(0)$, taken over the spatial index. For multiple different particle populations we use a different definition of $\Omega$; see Ref. [14] for details. Further, we define a new random variable $\eta_{i}$ (which is of order one as $\Omega \rightarrow \infty$ ), through the relation

$$
U_{i}=\phi_{i} \Omega+\eta_{i} \sqrt{\Omega}
$$

where $\phi_{i}$ is the expected ratio of population at time $t$ to the initial population. Finally, we link the statistics of $U_{i}$ and $\eta_{i}$ through the identification [15]

$$
P(\boldsymbol{U}, t)=\Pi(\eta, t) .
$$

Using Taylor series, at order $\sqrt{\Omega}$ we obtain [14]

$$
\sum_{i=1}^{K} \frac{d \phi_{i}}{d t} \frac{\partial \Pi}{\partial \eta_{i}}=\sum_{i=1}^{K} \sum_{j=1}^{J} \mathrm{a}_{j}(\boldsymbol{\phi}) v_{i j} \frac{\partial \Pi}{\partial \eta_{i}},
$$

where $\mathrm{a}_{j}$ is the macroscopic propensity of reaction $j$. At order one we derive a Fokker-Planck equation [22], which defines the dynamics of the probability density,

$$
\frac{\partial \Pi}{\partial t} \stackrel{\text { def }}{=}-\sum_{i, l=1}^{K} A_{i l} \frac{\partial\left(\eta_{l} \Pi\right)}{\partial \eta_{i}}+\frac{1}{2} \sum_{i, l=1}^{K} B_{i l} \frac{\partial^{2} \Pi}{\partial \eta_{i} \partial \eta_{l}},
$$

where [23]

$$
\begin{aligned}
\boldsymbol{A} & =\left\{A_{i l}\right\}=\left\{\partial[\boldsymbol{v a}]_{i} / \partial \phi_{l}\right\}, \\
\boldsymbol{B} & =\left\{B_{i l}\right\}=\boldsymbol{v} \operatorname{diag}(\mathbf{a}) \boldsymbol{v}^{T}, \\
\boldsymbol{v} & =\left\{v_{i j}\right\}_{i=1, \ldots, K}^{j=1, \ldots, J},
\end{aligned}
$$

and $\mathrm{a}^{T}=\left(\mathrm{a}_{1}, \ldots, \mathrm{a}_{J}\right)$.

\section{B. Spatially extended systems}

Although other descriptions are possible [24], here we model diffusion as a space-jump process [13] (see Fig. 1). As mentioned in Sec. II, since the chemical population inhabits a one-dimensional domain of length $L$ we discretize the population and domain into $K$ compartments of size $\Delta_{E}=L / K$. 


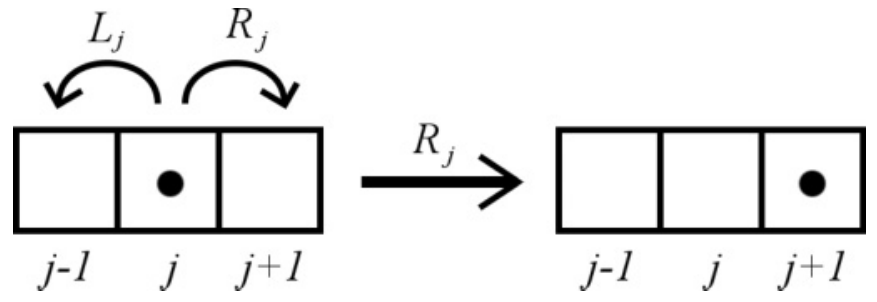

FIG. 1. Diagram of the space-jump description of diffusion. Each particle has equal probability of moving left as of moving right. If, for example, the right diffusion reaction $R_{j}$ occurs one molecule from box $j$ moves to box $j+1$. Reproduced from Ref. [14].

The space-jump description of diffusion is then defined by the reactions

$$
S_{1} \underset{d_{E}}{\stackrel{d_{E}}{\rightleftharpoons}} S_{2} \underset{d_{E}}{\stackrel{d_{E}}{\rightleftharpoons}} \cdots \underset{d_{E}}{\stackrel{d_{E}}{\rightleftharpoons}} S_{K},
$$

where $d_{E}$ is the microscopic diffusion coefficient. Note that we use Neumann boundary conditions throughout this paper, and, so, particles do not flow out of the domain.

Since these are first-order equations, the equations governing their mean values are equivalent to the deterministic reactions [25]. It is seen that if $d_{E}=D / \Delta_{E}^{2}$, where $D$ is the macroscopic rate of diffusion [26], then the mean-field equations are equivalent to a second-order finite difference approximation of the one-dimensional Laplacian, which is derived through Taylor expansions of the continuous formulation [27]. Thus, the expected value of the populations undergoing stochastic diffusion corresponds to the deterministic diffusion equation in the limit $\Delta_{E} \rightarrow 0$.

Using the matrix terminology developed in Sec. II A, we calculate the stoichiometric matrix, $v$, and the macroscopic transition rate, $a$, from the reactions in (9). These can then be used to inform the definitions (6) and (7) and show that $\boldsymbol{A}$ and $\boldsymbol{B}$ have symmetric tridiagonal forms. It is these key features that allow us to derive their spatial Fourier transforms. In Sec. IV, we will extend our considerations to pentagonally banded matrices.

\section{Spatial Fourier transform}

We now introduce the discrete spatial Fourier transform [28-31]. We choose to use the discrete Fourier cosine expansion $[14,30,32,33]$, since, by setting $k=m \pi / L, m=$ $0,1, \ldots, K-1$, we can incorporate the boundary terms as the cosine function naturally encapsulates the Neumann boundary conditions. The transform has the explicit form

$$
\widehat{f}(k)=\Delta_{x} \sum_{j=1}^{K} \cos \left[k \Delta_{x}(j-1)\right] f\left(x_{j}\right) .
$$

Note that the term $(j-1)$ in the cosine function is simply to correct for the fact that, spatially, we have defined our populations to start with an index one instead of zero.

\section{LAGRANGIAN COORDINATES}

In transforming to the Lagrangian domain we move from considering the microscopic activities of each particle to a mesoscopic formulation. This is the key step that allows us to develop a description of growth that can be rigorously linked to the macroscopic equation (see Ref. [14]). Although we have changed the scale at which we are viewing the population, the diffusion is still a space-jump process on this Lagrangian discretized domain. However, once we include growth, the diffusion rate is no longer a constant. We start by discretizing the domain into $K$ compartments, as usual. These boxes, of size $\Delta_{L}=L / K$, are now identified to be the Lagrangian compartments, and each compartment $i$ is assumed to initially contain $N_{i}(0)$ Eulerian subcompartments of size $\Delta_{E}$, and thus $\Delta_{L}=N_{i}(0) \Delta_{E}$. This microscopic variable $N_{i}(t)$ is then also controlled stochastically and is identified, as in Sec. II A, as a linear combination of a deterministic part $n_{i}$, which defines the average ratio of the total size of the corresponding Eulerian subcompartments, $N_{i}(t) \Delta_{E}$, at a time $t$ to Lagrangian box size $\Delta_{L}$ and a stochastic part $\epsilon_{i}(t)$ through

$$
N_{i}=n_{i} \theta+\epsilon_{i} \sqrt{\theta},
$$

where

$$
\theta \stackrel{\text { def }}{=} \frac{\Delta_{L}}{\Delta_{E}}
$$

is the scaling of Eulerian boxes to Lagrangian box length. Initially, the $N_{i}(0)$ are taken to be equal for all $i$; thus,

$$
\Delta_{L} n_{i}(0)=\theta \Delta_{E}=N_{i}(0) \Delta_{E},
$$

(see Fig. 2), and hence $n_{i}(0)=1$ for all $i$.

Similar to the Eulerian description in Sec. II A, we link the random variables $\boldsymbol{N}=\left(N_{1}, \ldots, N_{K}\right)$ and $\boldsymbol{U}=\left(U_{i}, \ldots, U_{K}\right)$ to $\boldsymbol{\epsilon}=\left(\epsilon_{1}, \ldots, \epsilon_{K}\right)$ and $\boldsymbol{\eta}=\left(\eta_{1}, \ldots, \eta_{K}\right)$ through the identification

$$
P(\boldsymbol{U}, \boldsymbol{N}, t)=\Pi(\boldsymbol{\eta}, \boldsymbol{\epsilon}, t),
$$

where $P(\boldsymbol{U}, \boldsymbol{N}, t)$ is, once again, the probability of being in state $(\boldsymbol{U}, \boldsymbol{N})$ at time $t$.

In the deterministic setting, we have shown that the effect of mapping a growing domain to a static one is simply realized as a scaling of the diffusion coefficient $[8,14]$. On expanding in the weak noise limit the leading-order term is the deterministic equation; hence we require a stochastic

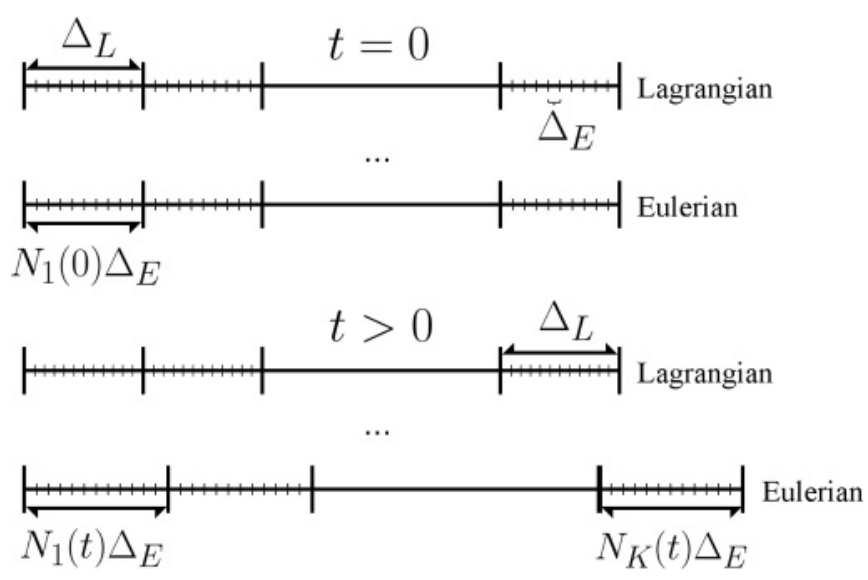

FIG. 2. Illustrating the connection between the Lagrangian description of the domain and the corresponding Eulerian description. See text for details. Reproduced from Ref. [14]. 
domain growth generalization of the jump rates, which in the deterministic growth scenario are given by $d_{L}=D / \Delta_{L}^{2}$.

The stochastic effects of the domain are then added through noting that

$$
\lim _{\theta \rightarrow \infty} \frac{N_{i}(t)}{\theta}=n_{i}(t)
$$

and substituting $n_{i}(t)$ for $N_{i} / \theta$. However, since the growth is uniform, all of the Eulerian subdivisions, $N_{i}$, of a Lagrangian box $i$ are controlled identically, and so, in the mean field, the $n_{i}(t)$ will be equal for all $i$. Thus, many forms of dependence of the diffusion coefficient on the spatial variables will give rise to equivalent forms of mean-field growth. Two particular justified examples are considered in the next section.

Presently, we continue with a general diffusion coefficient $d_{i, j}$, which is the transition rate from Lagrangian box $i$ to the Lagrangian box $j$ such that $d_{i, j} \rightarrow d_{L} / n(t)^{2}$ as $\theta \rightarrow \infty$. Although $d_{i, j}$ is the transition rate between boxes $i$ and $j$ we do not explicitly define its dependence on the Eulerian populations. In particular, we do not know if the description is symmetric; i.e., $d_{i, j}$ may not be equal to $d_{j, i}$.

By suppressing the unchanged variables, the CME describing diffusion in the Lagrangian framework, on a stochastically growing domain, has the general form

$$
\begin{aligned}
\frac{\partial P}{\partial t}= & \sum_{i=1}^{K-1} d_{i, i+1}\left[\left(U_{i}+1\right) P\left(\ldots, U_{i}+1, U_{i+1}-1, \ldots\right)-U_{i} P\right]+\sum_{i=2}^{K} d_{i, i-1}\left[\left(U_{i}+1\right) P\left(\ldots, U_{i-1}-1, U_{i}+1, \ldots\right)-U_{i} P\right] \\
& +\sum_{i=1}^{K} G_{i}\left(N-\boldsymbol{v}_{i}\right) P\left(N-\boldsymbol{v}_{i}\right)-G_{i}(\boldsymbol{N}) P .
\end{aligned}
$$

The first two lines of the summation describe right and left jumps, respectively. The last line defines growth through the increase in the number of subdivisions of each compartment, which feeds back to the diffusion terms through the dependence of the $d_{i, j}$ on $N_{i}$. In Eq. (16) the form of growth is left general through the use of the operator $G_{i}$. We now specify the growth to be uniformly linear with rate $r$; i.e., the rate of increase in subdivisions $N_{i}$ of Lagrangian box $i$ is constant over time, and hence, $G_{i}(N)=r / \theta$. This is easily modified mutatis mutandis.

Approaching the CME through the formalism of van Kampen's linear noise expansion method [15] gives

$$
\begin{aligned}
\frac{\partial \Pi}{\partial t} & -\sqrt{\Omega} \sum_{i=1}^{K} \frac{\partial \Pi}{\partial \eta_{i}} \frac{d \phi_{i}}{d t}-\sqrt{\theta} \sum_{i=1}^{K} \frac{\partial \Pi}{\partial \epsilon_{i}} \frac{d n_{i}}{d t} \\
= & \sum_{i=1}^{K-1} d_{i, i+1} \frac{1}{\sqrt{\Omega}}\left(\frac{\partial}{\partial \eta_{i}}-\frac{\partial}{\partial \eta_{i+1}}\right)\left(U_{i} \Pi\right)+\frac{1}{2 \Omega} \sum_{i=1}^{K-1} d_{i, i+1}\left(\frac{\partial}{\partial \eta_{i}}-\frac{\partial}{\partial \eta_{i+1}}\right)^{2}\left(U_{i} \Pi\right)+\sum_{i=1}^{K-1} d_{i+1, i}\left(-\frac{\partial}{\partial \eta_{i}}+\frac{\partial}{\partial \eta_{i+1}}\right)\left(U_{i+1} \Pi\right) \\
& +\frac{1}{2 \Omega} \sum_{i=1}^{K-1} d_{i+1, i}\left(-\frac{\partial}{\partial \eta_{i}}+\frac{\partial}{\partial \eta_{i+1}}\right)^{2}\left(U_{i+1} \Pi\right)-\sum_{i=1}^{K} \frac{1}{\sqrt{\theta}} \frac{r}{\theta} \frac{\partial \Pi}{\partial \epsilon_{i}}+\frac{1}{2 \theta} \frac{r}{\theta} \sum_{i=1}^{K} \frac{\partial^{2} \Pi}{\partial \epsilon_{i}^{2}}
\end{aligned}
$$

Since

$$
\frac{N_{i}}{\theta}=n_{i}+\frac{\epsilon_{i}}{\sqrt{\theta}} \rightarrow n_{i} \quad \theta \rightarrow \infty,
$$

we use the approximation

$$
\frac{1}{n_{i}(t)+\frac{\epsilon_{i}(t)}{\sqrt{\theta}}} \approx \frac{1}{n_{i}(t)}-\frac{\epsilon_{i}}{\sqrt{\theta} n_{i}(t)^{2}}+\frac{\epsilon_{i}^{2}}{\theta n_{i}(t)^{3}} .
$$

This approximation allows us to explicitly consider the various orders of $\theta$.

It should be noted that Eq. (19) holds only when $\left|\epsilon_{i}(t)\right| \ll$ $\sqrt{\theta} n(t)$. Since we are using a weak expansion of the noise, which was motivated by the central limit theorem, we assume that, at leading order, the noise will be Gaussian and able to take values of arbitrary size [15]. However, $\left\langle\epsilon_{i}\right\rangle=0$ and initially $\left\langle\epsilon_{i}^{2}\right\rangle=0$, since the starting conditions are assumed to be known. Through the Fokker-Planck equation, generated from the linear noise expansion, we are able to derive expressions for the evolution of the variance. Thus, we can put probabilistic bounds on how likely Eq. (19) is to hold at a certain time. This is done for the examples considered in Sec. V.
Derivatives with respect to $\epsilon_{i}$ decouple from Eq. (17) and thus to first order in $\sqrt{\theta}$,

$$
\dot{n}_{i}=\frac{r}{\theta} \quad \text { for } \quad i=1, \ldots, K .
$$

Thus, $n_{i}(t)=n(t)=1+r t / \theta$, using the initial condition $n_{i}(0)=1$. Similarly, by using Eq. (19), we can equate leading orders of $\sqrt{\Omega}$ to obtain

$$
\begin{gathered}
\frac{\partial \phi_{1}}{\partial t}=\frac{d_{L}}{n^{2}}\left(\phi_{2}-\phi_{1}\right), \\
\frac{\partial \phi_{i}}{\partial t}=\frac{d_{L}}{n^{2}}\left(\phi_{i+1}-2 \phi_{i}+\phi_{i-1}\right) \quad \text { for } \quad i=2, \ldots, K-1, \\
\frac{\partial \phi_{K}}{\partial t}=\frac{d_{L}}{n^{2}}\left(\phi_{K-1}-\phi_{K}\right) .
\end{gathered}
$$

Remembering that $n(t)=1+r t / \theta$ and defining $d_{L}=D / \Delta_{L}^{2}$, where $D$ is the macroscopic diffusivity, we can see that, on taking $K \rightarrow \infty$ or, equivalently, $\Delta_{L} \rightarrow 0$, we retrieve the discretized diffusion PDEs mapped from the growing Eulerian domain onto a stationary Lagrangian domain. 

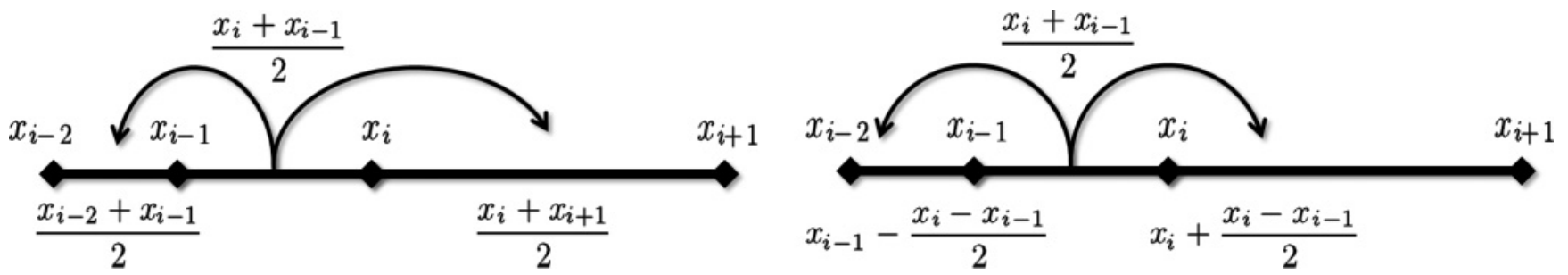

FIG. 3. Illustration of different jumping paradigms. Note that initially each Lagrangian compartment will be the same size, but, due to stochastic growth, it is highly likely that the boxes will be perturbed away from the average length. Thus, the small, medium, and large compartment sizes are for illustrative purposes only. See text for details.

Having removed the leading order equations of $\Omega$ and $\theta$ we obtain terms of order $\sqrt{\Omega / \theta}$, which are exactly the terms that would arise from using Elf and Ehrenberg's matrix method [23], discussed in Sec. II A. The only generalization is that the scaling factors, which normally cancel, remain. Thus the general Fokker-Planck equation for a diffusing species on a stochastically growing domain is

$$
\frac{\partial \Pi}{\partial t}=-\sum_{i, j=1}^{2 K} A_{i j} \frac{\partial}{\partial \zeta_{i}}\left(\zeta_{j} \Pi\right) \sqrt{\frac{\Theta_{j}}{\Theta_{i}}}+\frac{1}{2} \sum_{i, j=1}^{2 K} B_{i j} \frac{\partial^{2} \Pi}{\partial \zeta_{i} \partial \zeta_{j}} \frac{\Theta_{j}}{\Theta_{i}}
$$

where $\Theta_{i}$ is the scaling for the variable $\zeta_{i}$. In the current case $\zeta_{i}=\eta_{i}$ and $\Theta_{i}=\Omega$ for $i=1, \ldots, K$, while $\zeta_{i}=\epsilon_{i}$ and $\Theta_{i}=\theta$ for $i=K+1, \ldots, 2 K$.

If $\theta \gg \Omega$ growth may be modeled deterministically. This case is considered in the previous paper [14]. Conversely, if $\theta \ll \Omega$ the noise generated through growth is far more important than that generated through population fluctuations. This case is not considered, since, biologically, we expect to be dealing with low population copy numbers. For simplicity, when stochastic growth is considered, we fix this ratio of nondimensionalized parameters to be one. In this case stochastic effects from domain growth and the diffusing populations are of the same order and are, thus, important.

Currently, we are unable to link the Lagrangian densities to the Eulerian description since we assume that the particle population within a Lagrangian box is uniformly distributed. However, although we cannot directly link populations to specific Eulerian compartments, we can link them to the large groups of Eulerian compartments that make up the Lagrangian compartments. Ultimately, because we can only rigorously link the meso- and macroscopic scale, our interpretation of the patterns must remain on this scale.

\section{DIFFERENT GROWING DOMAIN DESCRIPTIONS}

Growth has previously been defined through a box-splitting operation on the populations [13]. Initially, if an interval $[0, L]$ is split into $K$ boxes of length $L / K$, and the growth "reaction" occurs in the $j$ th box, a new box of size $L / K$ is created next to the $j$ th box, and the parent population is split between these two new daughter compartments. Various splitting rules have been used, including splitting the population uniformly randomly over the two boxes; however, these have been seen to have little effect on the stochastic simulations.
However, although microscopically this is a description of growth, the resulting CME that defines the operation does not have a weak noise limit, which is why we have constructed the Lagrangian domain. Due to this lack of a link between the scales, we cannot derive the spatial dependence of the Lagrangian transition rates from the Eulerian diffusion transition rates. In our previous paper [14], the specific dependence of the space-jump process on the discretization of the domain did not matter as the growth was deterministic, and, thus, the Lagrangian compartments were identical. In this section, and in the Appendix, we will show that the dependence of the diffusion coefficient on the spatial variables can have important consequences for the outcome of the simulations, which can be characterized by the different shapes of their power spectra.

Since there is currently a nonuniqueness in the transition rate description we will consider the following two types of Lagrangian diffusion (see Fig. 3 for a schematic diagram of the two types):

1. Jumping from the midpoint of a Lagrangian box to the midpoint of an adjoining Lagrangian box. We call this midpoint-to-midpoint diffusion (MMD). The justification for this description of diffusion is that we expect the particles to be uniformly distributed, due to the well-mixed assumption. Thus, we suppose that we can model diffusion as jumping between the Lagrangian centroids, due to the symmetry of density about this point.

2. Jumping a distance equal to the current Lagrangian box length from the midpoint of the Lagrangian box to an adjoining Lagrangian box. We call this approximate midpointto-midpoint diffusion (AMMD). The justification of this is that, since growth is uniform on average, the $N_{i}$ should all be approximately equal, given an homogeneous initial condition. Thus moving a distance equal to the Lagrangian box from the center of the box would move the particle to approximately the center of the adjoining Lagrangian box.

Note that we are not saying that AMMD is a better description of diffusion than MMD, which is a natural description to use. We use AMMD simply because it is a different form of transition rate dependence that gives different effects, even though it should approximate the action of MMD.

From the Appendix we find that we can calculate the transition rates from box $i$, to the left, $l_{i}$ and to the right, $r_{i}$. These have the explicit forms

$$
\begin{aligned}
l_{i} & =\frac{8 d_{L}}{\frac{1}{\theta^{2}}\left(N_{i}+N_{i-1}\right)\left(N_{i-1}+2 N_{i}+N_{i+1}\right)}, \\
r_{i} & =\frac{8 d_{L}}{\frac{1}{\theta^{2}}\left(N_{i}+N_{i+1}\right)\left(N_{i-1}+2 N_{i}+N_{i+1}\right)},
\end{aligned}
$$


for MMD and

$$
\begin{aligned}
& l_{i}=\frac{d_{L}}{\frac{1}{\theta^{2}} N_{i}^{2}}, \\
& r_{i}=\frac{d_{L}}{\frac{1}{\theta^{2}} N_{i}^{2}},
\end{aligned}
$$

for AMMD.

Since $N_{i}=\theta n_{i}+\sqrt{\theta} \epsilon_{i}$, we see that either of the transition rates will give rise to our mean-field description of diffusion on a growing domain as $\theta \rightarrow \infty$. Although these two descriptions are equivalent in the limit of large $\theta$, Fig. 4 shows that AMMD leads to more consistent local maxima and minima than MMD; i.e., stripes, which are not apparent in Figs. 4(a) and 4(c), are visible in Figs. 4(b) and 4(d).

These extrema form under Eqs. (27) and (28) and not Eqs. (25) and (26) due to the different dependencies on the spatial variables. Notice in MMD that the effect of having a local extremum of Eulerian subdivisions $N_{i}$ is reduced. This is due to the local number of subdivisions being averaged against the number of Eulerian subdivisions in the adjacent boxes, $N_{i-1}$ and $N_{i+1}$. However, now consider AMMD and suppose a particular Lagrangian compartment $i$ contains a local maximum of Eulerian subdivisions $N_{i}$. By assumption, the transition rate out of this compartment will be smaller than the transition rates into the compartment, i.e.,

$$
\text { r } \begin{aligned}
\frac{d_{L}}{N_{i}(t)^{2} / \theta^{2}} & <\frac{d_{L}}{N_{i+1}(t)^{2} / \theta^{2}}, \\
\text { and } \frac{d_{L}}{N_{i}(t)^{2} / \theta^{2}} & <\frac{d_{L}}{N_{i-1}(t)^{2} / \theta^{2}} .
\end{aligned}
$$

Hence, more particles flow into the compartment producing a local maximum of the population, $U_{i}$. Further, this maximum will last as long as the compartment contains a local maximum of Eulerian compartments. Conversely, if the Eulerian population is a local minimum, the transition rates out of the compartment will be higher than the transition rates into the compartment. Thus, the compartment will tend to a population minimum.

As a final comment we emphasize that this heterogeneity is not simply due to the description of diffusion we are using. The stochasticity is critical in the nucleation of the stripes, as it is the varying number of subdivisions that creates the local extrema. This, in turn, reinforces the asymmetry of the descriptions, as shown in Eq. (29). In using the weak noise expansion we assume that the population of Eulerian boxes per Lagrangian box is large enough such that the stochastic effects are of lower order, and, thus, the size of compartments should differ only by a small amount. Hence, we are illustrating that these small differences on the Eulerian scale can have large effects on the Lagrangian population.

When we were considering the basic case of our stochastic description of diffusion on a static, or deterministically growing, domain the matrices $\boldsymbol{A}$ and $\boldsymbol{B}$ were $K \times K$ tridiagonal. Now, we have the additional population of Lagrangian subdivisions $N_{i}$; thus, the matrices will be $2 K \times 2 K$ in size. Generally, they will be $p K \times p K$ in size, where $p$ is the number of distinct, spatially extended, reacting species. This $2 K \times 2 K$ (more generally $p K \times p K$ ) matrix can be split up into $K \times K$ submatrices, which can be linked to the covariances of each of the species, with each submatrix having strong diagonal features. These diagonal features are discussed in the following section.

\section{FOURIER ANALYSIS OF ADDITIONAL REACTIONS}

In this section we methodically apply the developed Fourier techniques to the case of MMD on a linearly growing domain, with rate of growth $r$. The case of AMMD is similar and discussed later. First, we define the stoichiometric matrix and the macroscopic propensity functions:

$$
\begin{aligned}
& \text { Right jump Left jump Growth } \\
& \left(\begin{array}{ccccc|ccccc|cccc}
-1 & 0 & \cdots & 0 & 0 & 0 & 1 & 0 & \cdots & 0 & 0 & \cdots & \cdots & 0 \\
1 & \ddots & \ddots & \vdots & \vdots & \vdots & -1 & \ddots & \ddots & \vdots & \vdots & \ddots & \ddots & \vdots \\
0 & \ddots & \ddots & 0 & \vdots & \vdots & 0 & \ddots & \ddots & 0 & \vdots & \ddots & \ddots & \vdots
\end{array}\right) \quad \text { Effect on diffusing population, }
\end{aligned}
$$

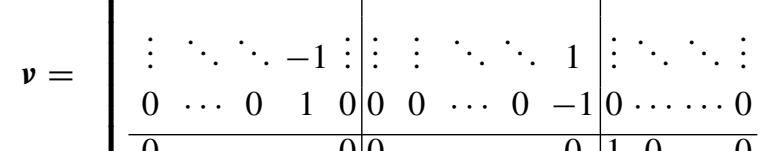

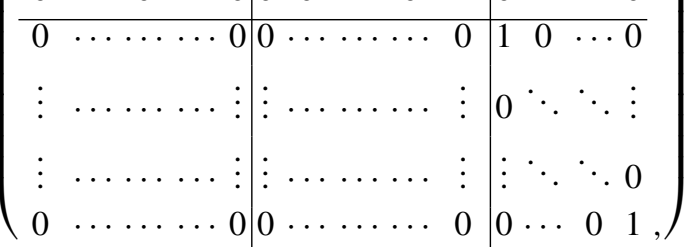

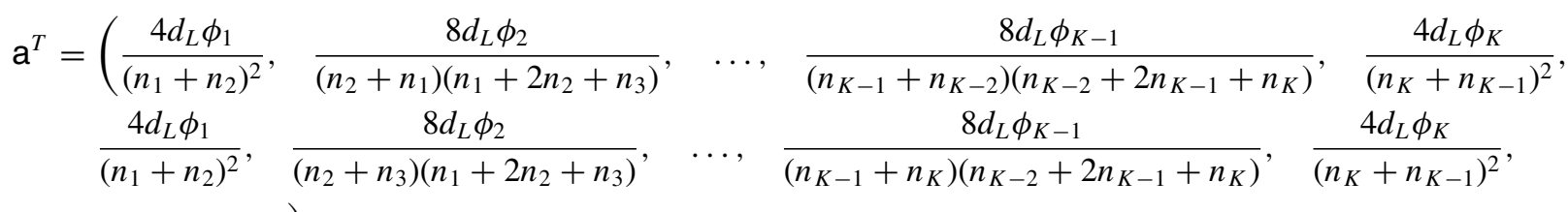

$$
\begin{aligned}
& \left.\frac{r}{\theta}, \ldots, \frac{r}{\theta}\right) \text {. }
\end{aligned}
$$




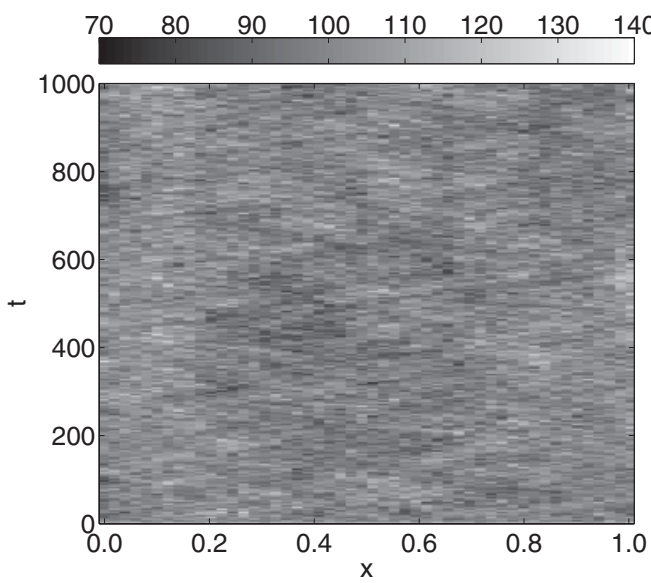

(a) $r=0.01$.

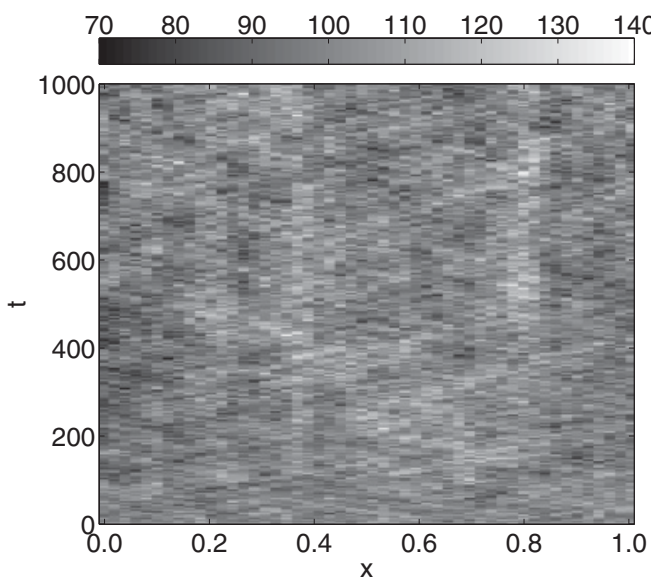

(c) $r=0.1$.

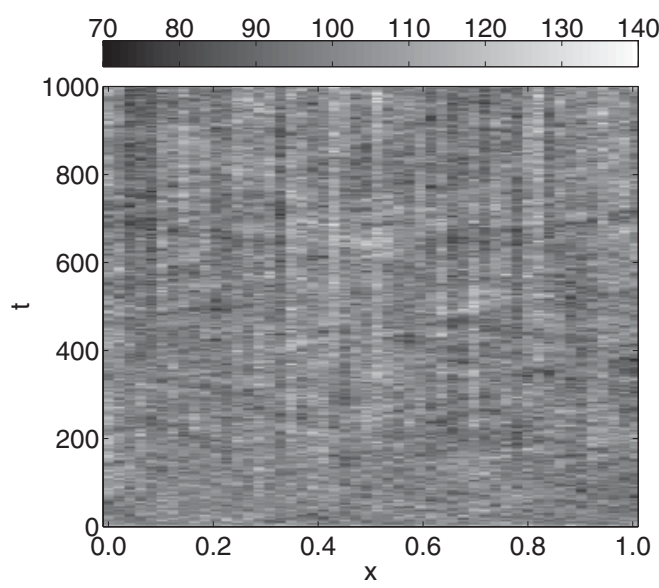

(b) $r=0.01$.

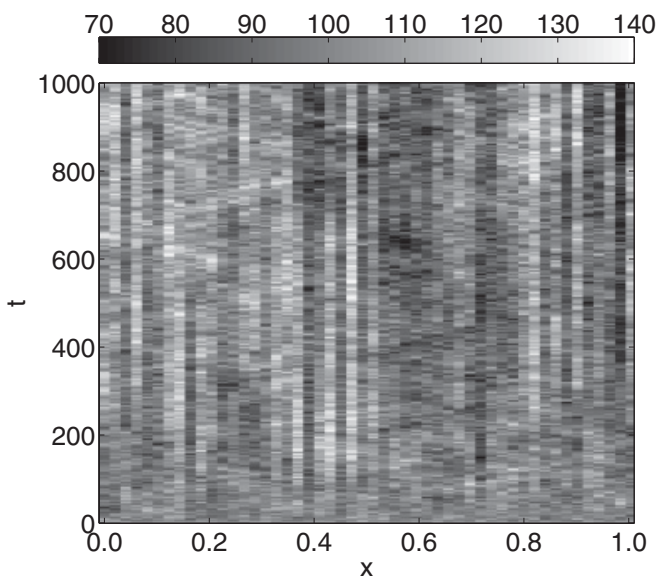

(d) $r=0.1$.

FIG. 4. Single realizations, comparing different forms of Lagrangian diffusion on linearly growing domains. Left column: MMD, the time dependence of the transition rate is defined by Eqs. (25) and (26). Right column: AMMD, the time dependence of the transition rate is defined by Eqs. (27) and (28). In each case the domain is growing linearly with rate $r$ given under each figure. Other parameters are $d_{L}=1, K=50$, and $\theta=100$.

The stoichiometric matrix has been split into six submatrices. Each submatrix is $K \times K$ in size, and three of these are nonzero. The first $K \times K$ elements are the population change vectors of transitions to the right. Similarly, the second submatrix is made up of the transition vectors of jumps to the left. The last nonzero submatrix controls the change of Eulerian subdivisions in each Lagrangian box. In this case we are dealing with linear growth of Eulerian populations with rate $r$. The zeros in the rest of the submatrices show that diffusion does not alter the number of Eulerian subdivisions, and, similarly, the Eulerian subdivisions do not affect the diffusing populations except through the transition rate dependencies.

The propensity vector has also been split into the three types of reactions in order to aid visualization. The first and second lines are the macroscopic propensities of jumps right and left. The final line contains the propensity functions describing linear growth. Note the scaling of $\theta$ is needed because linear growth is a zeroth-order reaction.

We can now calculate the matrices $\boldsymbol{A}$ and $\boldsymbol{B}$ using their definitions, Eqs. (6) and (7). The matrices will have the following forms:

$$
A=\left(\begin{array}{ll}
a & b \\
c & d
\end{array}\right), \quad B=\left(\begin{array}{ll}
\alpha & \beta \\
\beta & \gamma
\end{array}\right),
$$

where all the submatrices are symmetric. Further $\boldsymbol{c}=\boldsymbol{d}=$ $\boldsymbol{\beta}=\mathbf{0}, \boldsymbol{\gamma}=(r / \theta) \boldsymbol{I}$, where $\boldsymbol{I}$ is the $K \times K$ identity matrix, and $\boldsymbol{a}$ and $\boldsymbol{\alpha}$ are tridiagonal symmetric matrices with diagonal entries $a_{0}=-2 d_{L} / n^{2}, \alpha_{0}=-4 d_{L} \phi / n^{2}$, and off-diagonal entries $a_{1}=d_{L} / n^{2}, \alpha_{1}=2 d_{L} \phi / n^{2}$, respectively, where $n=$ $1+r t / \theta$.

The form of $\boldsymbol{b}$ is pentagonal symmetric. To see this, consider the $i$ th $(2<i<K-1)$ population, $U_{i}$. $U_{i}$ will depend on the transition of particles out of the $i$ th box, $l_{i}$ and $r_{i}$, as well as the transition of the particles from the $(i-1)$-th and $(i+1)$-th boxes into the $i$ th box, namely, $r_{i-1}$ and $l_{i+1}$. By considering Eqs. (25) and (26), we see that $l_{i+1}, l_{i}, r_{i}$ and $r_{i-1}$ will depend on the variables $n_{i-2}, n_{i-1}, n_{i}, n_{i+1}$, and $n_{i+2}$. Thus, when the derivative is taken with respect to each variable to form $\boldsymbol{A}$, there will be at most five nonzero terms, all symmetric about the diagonal. Fortunately, the sub- and superdiagonals of $\boldsymbol{b}$ reduce to zero. Hence, the only nonzero terms of $\boldsymbol{b}$ are the 
diagonal, second-sub-, and second-superdiagonal. The explicit form of $\boldsymbol{b}$ is

$$
\boldsymbol{b}=\left(\begin{array}{ccccccc}
\frac{d_{L} \phi}{4 n^{3}} & 0 & -\frac{d_{L} \phi}{4 n^{3}} & 0 & \cdots & \cdots & 0 \\
0 & \frac{d_{L} \phi}{4 n^{3}} & 0 & -\frac{d_{L} \phi}{4 n^{3}} & 0 & \cdots & 0 \\
-\frac{d_{L} \phi}{4 n^{3}} & 0 & \frac{d_{L} \phi}{2 n^{3}} & 0 & -\frac{d_{L} \phi}{4 n^{3}} & \ddots & \vdots \\
0 & \ddots & \ddots & \ddots & \ddots & \ddots & 0 \\
\vdots & \ddots & -\frac{d_{L} \phi}{4 n^{3}} & 0 & \frac{d_{L} \phi}{2 n^{3}} & 0 & -\frac{d_{L} \phi}{4 n^{3}} \\
0 & \cdots & 0 & -\frac{d_{L} \phi}{4 n^{3}} & 0 & \frac{d_{L} \phi}{4 n^{3}} & 0 \\
0 & \cdots & \cdots & 0 & -\frac{d_{L} \phi}{4 n^{3}} & 0 & \frac{d_{L} \phi}{4 n^{3}}
\end{array}\right) .
$$

Using these forms of $\boldsymbol{A}$ and $\boldsymbol{B}$ we are able to derive the corresponding general Fokker-Planck equation for the variables $\eta_{i}, \epsilon_{i}$, where

$$
\begin{gathered}
U_{i}=\Omega \phi_{i}+\sqrt{\Omega} \eta_{i}, \\
N_{i}=\theta n_{i}+\sqrt{\theta} \epsilon_{i} .
\end{gathered}
$$

Remember that, in order to simplify the algebra, we $\operatorname{set} \theta=\Omega$. The general Fokker-Planck equation is thus

$$
\begin{aligned}
\frac{\partial \Pi}{\partial t}= & -\sum_{i, j} \boldsymbol{a}_{i j} \frac{\partial}{\partial \eta_{i}}\left(\eta_{j} \Pi\right)-\sum_{i, j} \boldsymbol{b}_{i j} \frac{\partial}{\partial \eta_{i}}\left(\epsilon_{j} \Pi\right) \\
& -\sum_{i, j} \boldsymbol{c}_{i j} \frac{\partial}{\partial \epsilon_{i}}\left(\eta_{j} \Pi\right)-\sum_{i, j} \boldsymbol{d}_{i j} \frac{\partial}{\partial \epsilon_{i}}\left(\epsilon_{j} \Pi\right) \\
& +\frac{1}{2} \sum_{i, j} \boldsymbol{\alpha}_{i j} \frac{\partial^{2} \Pi}{\partial \eta_{i} \partial \eta_{j}}+\sum_{i, j} \boldsymbol{\beta}_{i j} \frac{\partial^{2} \Pi}{\partial \eta_{i} \partial \epsilon_{j}} \\
& +\frac{1}{2} \sum_{i, j} \boldsymbol{\gamma}_{i j} \frac{\partial^{2} \Pi}{\partial \epsilon_{i} \partial \epsilon_{j}} .
\end{aligned}
$$

From Eq. (36) we are able to derive the covariances [15]

$$
\begin{aligned}
\left\langle\eta_{l} \dot{\eta}_{m}\right\rangle= & \sum_{j} \boldsymbol{a}_{l j}\left\langle\eta_{j} \eta_{m}\right\rangle+\sum_{j} \boldsymbol{a}_{m j}\left\langle\eta_{l} \eta_{j}\right\rangle+\sum_{j} \boldsymbol{b}_{l j}\left\langle\epsilon_{j} \eta_{m}\right\rangle \\
& +\sum_{j} \boldsymbol{b}_{m j}\left\langle\eta_{l} \epsilon_{j}\right\rangle+\boldsymbol{\alpha}_{l m}, \\
\left\langle\eta_{l} \epsilon_{m}\right\rangle= & \sum_{j} \boldsymbol{a}_{l j}\left\langle\eta_{j} \epsilon_{m}\right\rangle+\sum_{j} \boldsymbol{b}_{l j}\left\langle\epsilon_{j} \epsilon_{m}\right\rangle+\sum_{j} \boldsymbol{c}_{m j}\left\langle\eta_{l} \eta_{j}\right\rangle \\
& +\sum_{j} \boldsymbol{d}_{m j}\left\langle\eta_{l} \epsilon_{j}\right\rangle+\boldsymbol{\beta}_{l m}, \\
\left\langle\epsilon_{l} \epsilon_{m}\right\rangle= & \sum_{j} \boldsymbol{c}_{l j}\left\langle\eta_{j} \epsilon_{m}\right\rangle+\sum_{j} \boldsymbol{c}_{m j}\left\langle\epsilon_{l} \eta_{j}\right\rangle+\sum_{j} \boldsymbol{d}_{l j}\left\langle\epsilon_{j} \epsilon_{m}\right\rangle \\
& +\sum_{j} \boldsymbol{d}_{m j}\left\langle\epsilon_{l} \epsilon_{j}\right\rangle+\boldsymbol{\gamma}_{l m},
\end{aligned}
$$

where $=\partial / \partial t$. Due to linearity and the highly-banded, symmetric nature of matrices $\boldsymbol{A}$ and $\boldsymbol{B}$ we are immediately able to take the Fourier cosine transform of these equations (see Ref. [14] for detailed derivations). Note that, although we have not considered the case of a pentagonal matrix explicitly, it is a simple extension to show that the resulting Fourier
TABLE I. Explicit forms of the Fokker-Planck coefficients.

\begin{tabular}{lcc}
\hline \hline & Midpoint to midpoint & Approximate midpoint to midpoint \\
\hline$a_{0}$ & $\frac{-2 d_{L}}{n^{2}}$ & $\frac{-2 d_{L}}{n^{2}}$ \\
$a_{1}$ & $\frac{d_{L}}{n^{2}}$ & $\frac{d_{L}}{n^{2}}$ \\
$b_{0}$ & $\frac{d_{L} \phi}{2 n^{3}}$ & $\frac{4 d_{L} \phi}{n^{3}}$ \\
$b_{1}$ & 0 & $\frac{-2 d_{L} \phi}{n^{3}}$ \\
$b_{2}$ & $\frac{-d_{L} \phi}{4 n^{3}}$ & 0 \\
$\alpha_{0}$ & $\frac{4 d_{L} \phi}{n^{2}}$ & $\frac{4 d_{L} \phi}{n^{2}}$ \\
$\alpha_{1}$ & $\frac{-2 d_{L} \phi}{n^{2}}$ & $\frac{-2 d_{L} \phi}{n^{2}}$ \\
$\gamma_{0}$ & $\frac{r}{\theta}$ & $\frac{r}{\theta}$ \\
\hline \hline
\end{tabular}

transform can be generalized to

$$
b_{0}+2 \cos \left(k \Delta_{E}\right) b_{1}+2 \cos \left(2 k \Delta_{E}\right) b_{2},
$$

where $b_{0}$ are the diagonal elements, $b_{1}$ are the super- and subdiagonal elements, and $b_{2}$ are the second-sub- and secondsuperdiagonal elements.

Using the forms of the various submatrices discussed above yields

$$
\begin{gathered}
\left\langle\widehat{\eta}_{k} \widehat{\vartheta}_{k}\right\rangle=\left(4 a_{1} \cos \left(k \Delta_{L}\right)+2 a_{0}\right)\left\langle\widehat{\eta}_{k} \widehat{\eta}_{k}\right\rangle+\left(4 b_{2} \cos \left(2 k \Delta_{L}\right)\right. \\
\left.+2 b_{0}\right)\left\langle\widehat{\eta}_{k} \widehat{\epsilon}_{k}\right\rangle+\frac{\Delta_{L}^{2} K}{2}\left(\alpha_{0}+2 \cos \left(k \Delta_{L}\right) \alpha_{1}\right) \\
\left\langle\widehat{\eta}_{k} \widehat{\epsilon}_{k}\right\rangle=\left(2 a_{1} \cos \left(k \Delta_{L}\right)+a_{0}\right)\left\langle\widehat{\eta}_{k} \widehat{\epsilon}_{k}\right\rangle \\
+\left(2 b_{2} \cos \left(2 k \Delta_{L}\right)+b_{0}\right)\left\langle\widehat{\epsilon}_{k} \widehat{\epsilon}_{k}\right\rangle \\
\left\langle\widehat{\epsilon}_{k} \widehat{\epsilon}_{k}\right\rangle=\frac{\Delta_{L}^{2} K}{2} \gamma_{0}
\end{gathered}
$$

The case of AMMD can be treated similarly. The benefit of this different description of diffusion is that the transition probabilities depend only on $n_{i-1}, n_{i}$ and $n_{i+1}$, so the submatrix $\boldsymbol{b}$ is at most tridiagonal. Thus, it has an even simpler form than MMD.

\section{A. Power spectra comparison}

We now compare the derived descriptions of diffusion. In all cases the domain is growing linearly with rate $r$, and thus, $n=1+r t / \theta$.

Figures 5 and 6 compare the simulated and analytic spectra of the two descriptions. Notice that the simulations have been allowed to proceed until $t=1 \times 10^{5}$. Although the theory and simulation are still comparable, we should pay attention to the cautionary note in Sec. III on how long Eq. (19) stays valid. From Eq. (36) we see that the variance of linear growth is $\left\langle\epsilon_{i}(t)^{2}\right\rangle=r t / \theta$. As approximately $99.7 \%$ of the Gaussian distribution is between three standard deviations either side of the mean, then to have $99.7 \%$ of the data simulated correctly, we need to satisfy $3 \sqrt{\left\langle\epsilon_{i}(t)^{2}\right\rangle} \ll \sqrt{\theta} n(t)$. Expanding this implies

$$
\frac{r t / \theta}{(1+r t / \theta)^{2}} \ll \frac{\theta}{9} .
$$

Since $\theta$ is chosen to be large in order to satisfy the assumptions of the linear noise approximation, it follows that $\theta / 9 \gg 1$. Further, it can easily be shown that $u /(1+u)^{2} \leqslant 1 / 4$ for all $u \geqslant 0$, hence, the inequality holds for all $t \geqslant 0$. Thus, 


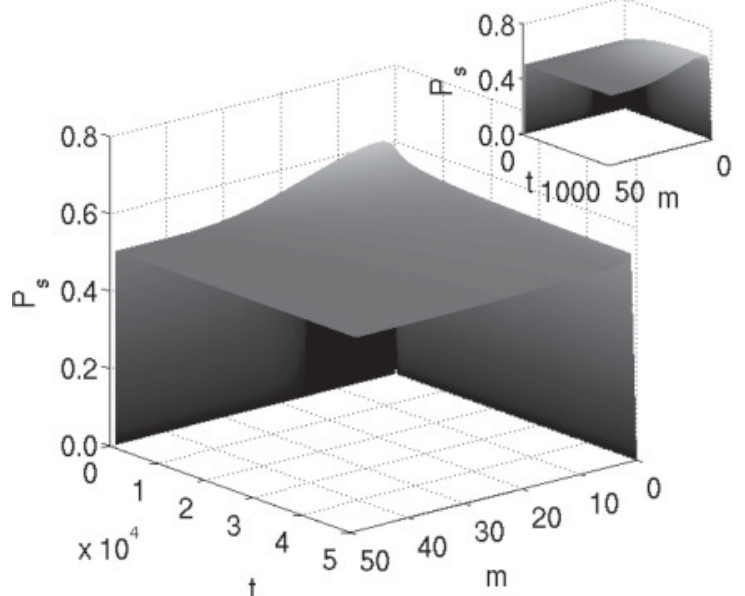

(a)

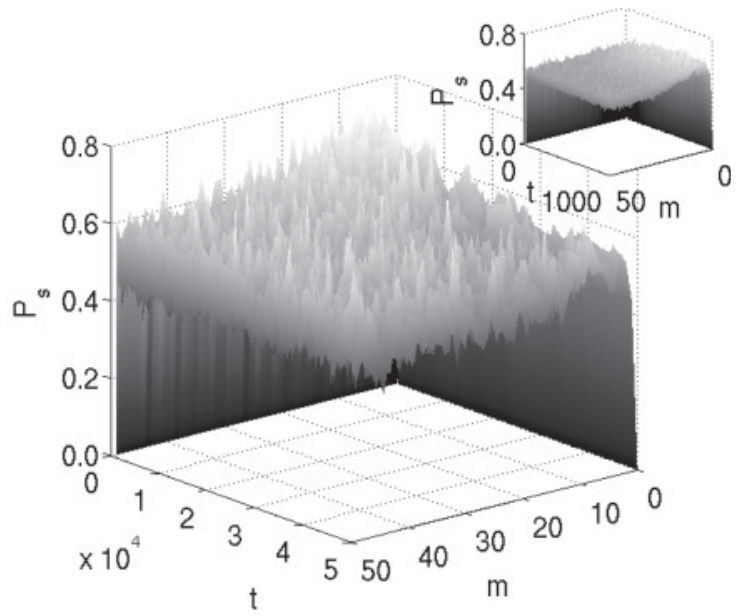

(b)

FIG. 5. Comparison of the theoretical power spectra (a), and the averaged power spectra of 200 simulations of MMD (b). Parameters are $r=0.1, \Delta_{L}=1 / 50, \Omega=\theta=100, k=m \pi / L, L=1$, and $d_{L}=1$. The inset in each figure shows a closeup of the first 1000 time units, and the stochastic spectrum is averaged over 1000 simulations. In each case the spectrum has been scaled by a factor of $\Delta_{L}^{2} K \phi$.

we expect the analysis to always be a good match for the simulation. However, although the analysis and simulations should always match, the physical interpretation of the domain discretization will eventually break down as it will become too coarse to accurately portray the spatial dynamics.

Not only do Figs. 5 and 6 show a good comparison between simulation and theory, but we also see differences between the two descriptions of diffusion. Both descriptions lead to an initial increase of power, above $P_{s}=0.5$. However, MMD excites only comparatively low wave modes, $m<30$, whereas AMMD excites all wave modes. In both simulations all wave modes, except $m=1$, relax to a steady state of $P_{s}=0.5$. For $m=1$ it should be noticed that AMMD has a higher value of power than MMD; however, overall, the stationary power spectra are very similar.

We can also compare these two types of stochastic growth with deterministic growth, Fig. 7, which was considered in
Ref. [14]. In the case of deterministic linear growth, the power spectrum is a monotonically increasing function of $k=\pi m / L$; hence we would never see the excited wave modes that occur in Figs. 5 and 6. Equally, as a function of time, the power spectrum is monotonic, and thus the initial increase past $P_{s}=0.5$ in the stochastic descriptions is never seen.

As noted, in the limit $r \rightarrow 0$ we regain the static domain equations; similarly, in the limit $\theta \gg \Omega$, the differences between the stochastic and deterministic formalisms disappear. However, within the region that the magnitudes of $\theta$ and $\Omega$ are comparable, we gain further evidence that noise can fundamentally affect systems in ways that are not considered through a purely deterministic description. Further, we conclude that although MMD and AMMD may be equivalent in the mean field, the internal noise generated by the descriptions of diffusion is able to affect the simulations in different ways.
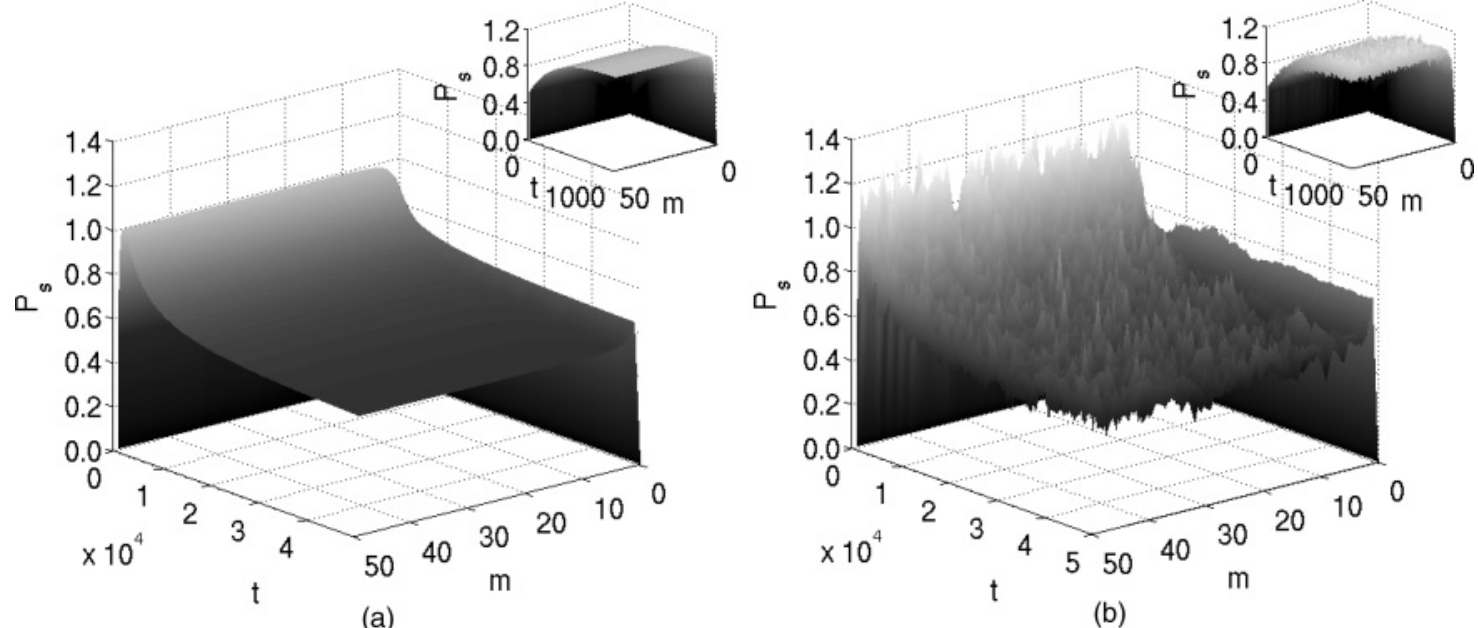

FIG. 6. Comparison of the theoretical power spectra (a), and the averaged power spectra of 200 simulations of AMMD (b). Parameters are $r=0.1, \Delta_{L}=1 / 50, \Omega=\theta=100, k=m \pi / L, L=1$, and $d_{L}=1$. The inset in each figure shows a closeup of the first 1000 time units, and the stochastic spectrum is averaged over 1000 simulations. In each case the spectrum has been scaled by a factor of $\Delta_{L}^{2} K \phi$. 


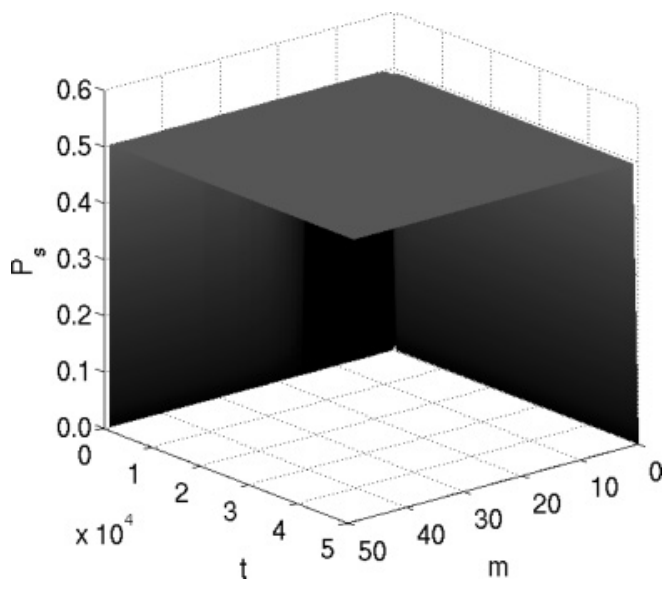

FIG. 7. Theoretical power spectra of diffusion on a deterministic linearly growing domain. Parameters are the same as in Fig. 6.

Hence, in order to simulate a system using a Lagrangian description correctly, we must be sure of the underlying stochastic description of movement. Finally, we comment on the issue that the time scale for the power spectra to reach their steady states is extremely long, approximately $2 \times 10^{4}$ time units. Mathematically this causes no problems; however, experimentally, the noise generated by growth on short time scales may be important since it is these initial effects that will determine the final inhomogeneities that arise. Thus, unless the growth of a diffusing system is adiabatically slow, the noise cannot be ignored.

\section{LOGISTICALLY GROWING DOMAIN}

As a final example, we consider the application of AMMD on a logistically growing domain,

$$
N_{i} \underset{b}{\stackrel{a}{\rightleftharpoons}} N_{i}+1 \text {. }
$$

The number of subdivisions, $N_{i}=\theta n_{i}+\sqrt{\theta} \epsilon_{i}$, in a Lagrangian box is controlled by the mean-field equation

$$
\dot{n}=a n-b \theta n^{2},
$$

and $K$ Langevin equations. These decouple and consist of $K$ identical equations of the form

$$
\dot{\epsilon}=-\left(a-2 b \theta n_{s}\right) \epsilon+\lambda,
$$

where $\langle\lambda, \lambda\rangle=a n_{s}+b \theta n_{s}^{2}$. Since $n_{s}=a /(b \theta)$, the variance of the random variable $\epsilon$ is

$$
\langle\epsilon(t) \epsilon(t)\rangle=\left(1-e^{-2 a t}\right) n_{s} .
$$

We now consider two sets of parameter values. These are $a=$ $1, b=10^{-2}$ and $a=10^{-3}, b=10^{-5}$. Since the value of $\theta$ is unchanged, the steady state $n_{s}$ is unchanged, and thus the long time steady state of the variance is the same. The difference between the two sets of coordinates is simply a matter of time scale. To transform the first set to the second set we use the transformation $t \mapsto t / 1000$. Based on this transformation, the first set of parameters is denoted fast, and the second set is denoted slow. The reason for this differentiation of the two scales can be seen by comparing Figs. 8(h) and 8(i). Although the (long-time, steady-state) variances of the two systems will be the same, the fast parameters cause perturbations about the mean to be faster, whereas the slow parameters produce a much weaker restorative force, and thus the stochastic trajectory is able to undertake extended periods away from the mean.

These two different scales are able to give very different forms of growth. Since we start the domain length at the meanfield steady state of Eq. (46), we expect the domain size not to alter very much; thus in Fig. 8 no domain growth (left column) is compared with quickly fluctuating logistic domain growth (middle column) and slowly fluctuating domain growth (right column). By following the routine set out in Sec. V we can construct the theoretical power spectrum for the logistically growing domain. Specifically, we are interested only in the steady state, which, for $k \neq 0$, is

$$
P_{s}(k)=\frac{\left\{\left(2 a d_{L} b^{2} \Omega^{2}+8 d_{L} \phi b^{3} \Omega^{3}\right)\left[\cos \left(k \Delta_{L}\right)-1\right]-a^{4}\right\}}{2 a\left\{2 d_{L} b^{2} \Omega^{2}\left[\cos \left(k \Delta_{L}\right)-1\right]-a^{3}\right\}} .
$$

Note that $P(0)=0$ since we subtract the expected value, and thus the constant mode, $k=0$, always has zero power. Again, we obtain excellent comparison between theory and simulation.

On comparing the power spectrum of diffusion on a slow logistically growing domain, Fig. 8(c), with diffusion on a static domain, Fig. 8(a), apart from the increase in power they appear very similar. However, when we compare actual diffusion simulations, fast logistic domain growth, Fig. 8(e), appears to be more similar to the stationary domain case, Fig. 8(d), than the highly patterned slow logistic growth, Fig. 8(f). Since the time scale of the deviations in the slow logistic growth is 1000 times slower than that for fast logistic growth, the slow-reacting domain is able to undergo longer periods away from the mean value, whereas the faster time scale of the middle column causes the system to rapidly correct itself whenever it overshoots the mean [compare Figs. 8(h) and 8(i)]. These extended meanderings enable the slower system to enter a patterned state; wherever the number of subdivisions in a Lagrangian box is higher than those surrounding it, the particles will diffuse slower out of the box than into it. Thus it is likely that this box will become a local maximum. Similarly, if a Lagrangian box has a lower number of subdivisions than those surrounding it, the diffusion out of this box will be faster than the diffusion into it, causing the box to become a local minimum. Thus, the longer the number of subdivisions of a Lagrangian box is perturbed away from the mean value, the higher the probability that the compartment will contain a local extremum of population. This then produces the patterned state that we see.

Hence, we have shown that, although we expect a homogeneous particle distribution on the logistically growing domain, growth is able to cause patterning, which is not possible in the deterministically growing case. Furthermore, the same form of growth can give rise to different effects depending on the time scale of the stochastic fluctuations.

\section{SUMMARY AND CONCLUSION}

In this paper we extended previous methods [14] to encompass the possibility of stochastic domain growth, which has led to the discovery that different Lagrangian transition 


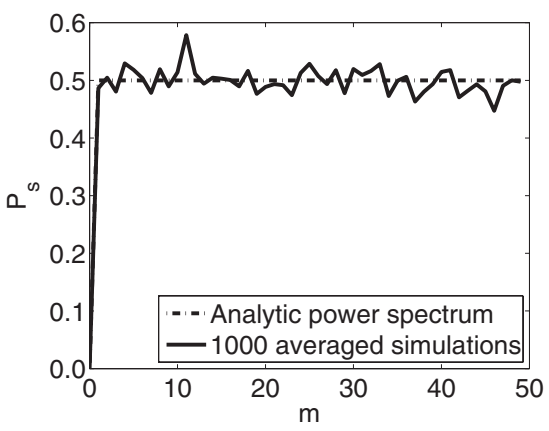

(a)

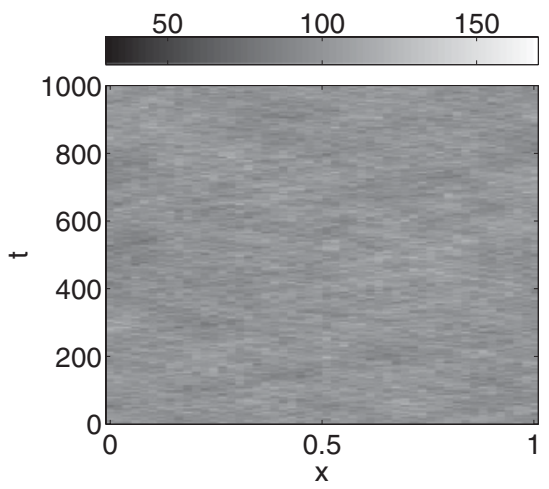

(d)

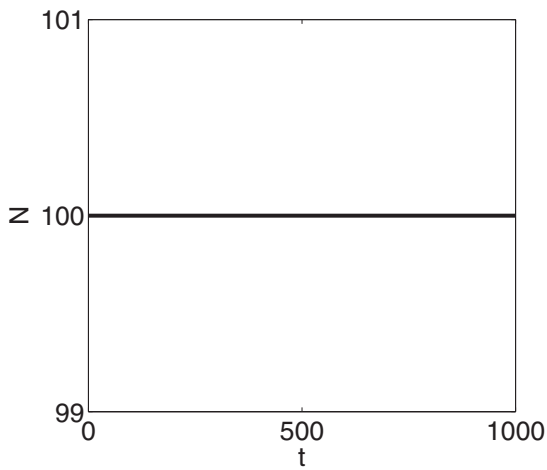

(g)

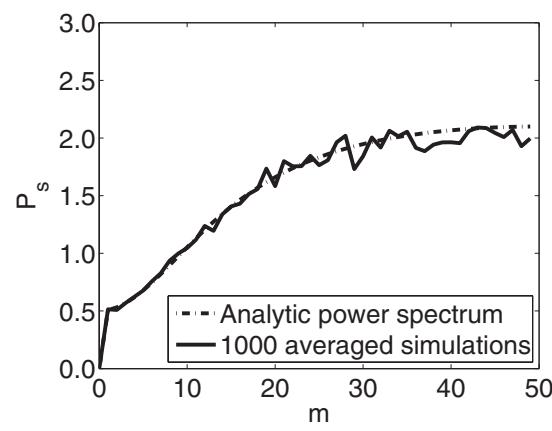

(b)

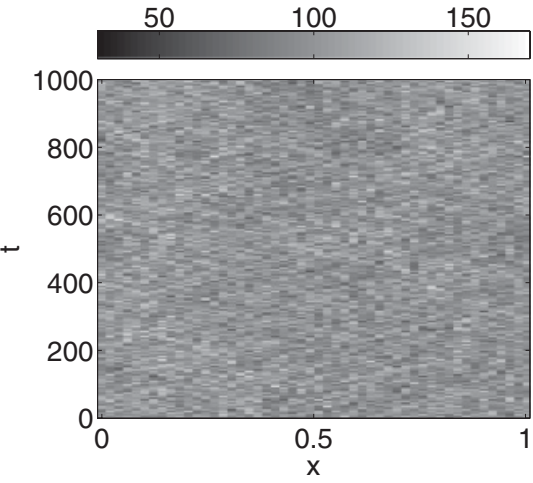

(e)

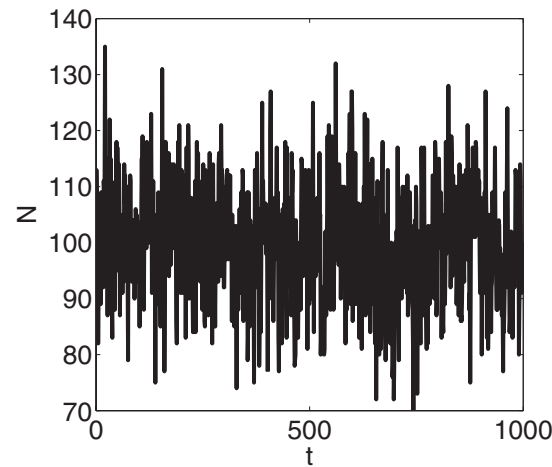

(h)

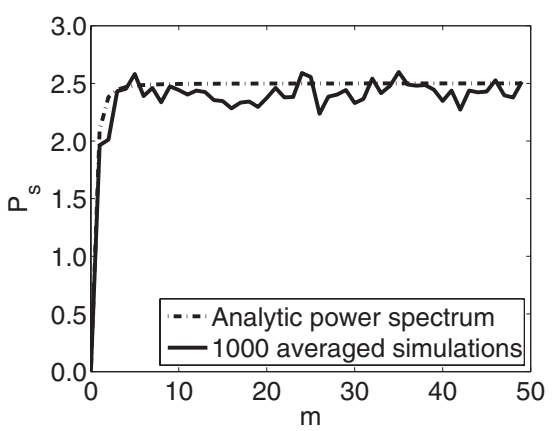

(c)

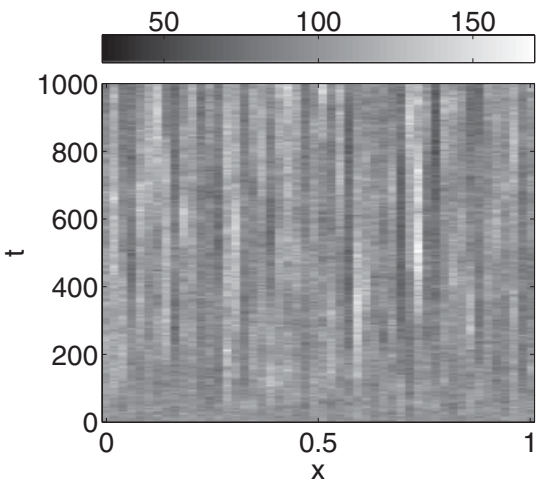

(f)

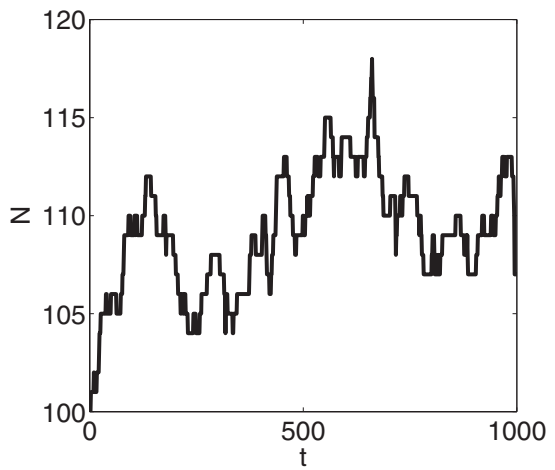

(i)

FIG. 8. Comparison of diffusion on a static domain with domains undergoing logistic growth with two different time scales. Left column: No growth. Middle column: Logistically growing domain with parameters $a=1$ and $b=1 / 100$. Right column: Logistically growing domain with parameters $a=10^{-3}$ and $b=10^{-5}$. Top row: Steady-state power spectrum of stochastic system averaged over 1000 trials, each scaled by $\Delta_{L}^{2} K \phi$ and compared with the theoretical steady-state power spectrum Eq. (49) similarly scaled. Middle row: Single realizations of the simulations. Bottom row: Single realizations of the number of subdivisions in the first Lagrangian box of the simulations in the middle row. Parameters $d_{L}=1, \Omega=\theta=100, \Delta_{L}=1 / 50, L=1, k=m \pi / L$, and $\phi=1$.

models can be proposed that each reduce to diffusion in the mean-field limit. Additionally, we have seen that stochastic effects are able to produce much richer dynamics than if a system had been treated as purely deterministic. Specifically, domain growth fundamentally changes the power spectrum of diffusion.

As mentioned, the most surprising result to come from the spatial mapping is that the definition of diffusion cannot be uniquely derived from the microscopic scale, due to the Lagrangian description of a growing domain. In order to specify an actual description of diffusion under Lagrangian growth, simulations were run using a variety of descriptions that are equivalent in the mean-field, such as those given in Eqs. (25)-(28). These were then compared with Eulerian box-splitting simulations that had been mapped back to the Lagrangian domain (data not shown). Although the steadystate power spectra were similar, there were fundamental differences in the evolution to the steady state. This is not surprising as currently there is no rigorous limit linking these two scales. Thus, although they are both descriptions of growth, only the Lagrangian formulation can be treated rigorously in a thermodynamic limit. However, this is not a big problem for the Lagrangian framework. It simply implies that, if we are to use the spatial mapping we must be clear about the underlying dependence of the motion on the domain. 
Due to the nonuniqueness of the description of diffusion, we considered two justified descriptions of diffusion on a Lagrangian growing domain: midpoint-to-midpoint diffusion (MMD) and approximate midpoint-to-midpoint diffusion (AMMD). Through simulations we have shown that the analysis compares favorably with Fourier transforming ensemble data. Further, at steady state, the power spectra are very similar. However, their respective evolutions to the steady state are completely different with AMMD exciting all wave modes to a higher power than MMD, which only, transiently, excited comparably low wave modes.

Physically, it should be noted that the Lagrangian description of growth becomes less valid as time increases; the domain is growing but the discretization remains fixed. If we were considering systems with spatial dynamics, we would have to take account of this by only allowing the system to evolve up until the point at which the Lagrangian discretization is too coarse to capture the relevant structures. However, although the physicality of the description becomes less valid, we have shown that we never need worry about the analytical approximations breaking down as we are able to simulate the data as accurately as we need, simply by increasing the parameter $\theta$ [see Eq. (44)].

These ideas were further extended to the example of diffusion on a logistically growing domain. Importantly, we saw the surprising result that the same type of growth can affect diffusion in different ways depending on the speed of correction of the stochastic perturbations. We concluded that there is a huge difference between a stationary domain and one that is stationary on average. Further, by inspection, the simulated results of diffusion on a stationary domain were much closer to the case of fast logistic kinetics, than slow logistic kinetics [see Figs. 8(d), 8(e), and 8(f)]. Although explainable, this is not necessarily an intuitive result as the slow logistic power spectrum qualitatively resembles the stationary domain case [see Figs. 8(a)-8(c)].

We have shown, analytically and computationally, that stochastic growth effects can play an important role when considering diffusing populations on a growing domain. Deterministically, the system will simply relax to a homogeneous steady state, whereas stochastic fluctuations in the underlying domain are able to cause nontransient structures to form, which have a wavelength that arises from a well-defined power spectrum. Importantly, we have demonstrated that this domain growth need not be monotonic; although deterministically logistic growth does produce monotonic growth to a final steady state, the intrinsic noise of the simulations causes the stochastic domain to oscillate about this mean-field with the interpretation that Eulerian subdivisions are being destroyed as well as created. We thus conclude that it is very important to clarify the dominant sources of noise, whether it be the population dynamics, growth or both, as the relationship between the respective noise scales, $\Omega$ and $\theta$, is able to produce large differences in the resulting power spectra.

Future work will apply this stochastic theoretical framework to biological patterning paradigms. Indeed, stochasticity has already been shown to extend the parameter regions over which Turing patterns are able to exist [33]. Previously, this small parameter region of existence has been seen to be one of the criticisms of using Turing systems as biological paradigms of pigmentation patterning [34]. We will further this reexamination of Turing systems by considering the effects of noise on the robustness of pattern generation [35].

\section{ACKNOWLEDGMENTS}

TEW would like to thank the Engineering and Physical Sciences Research Council for support. PKM was partially supported by the Royal Society Wolfson Foundation.

\section{APPENDIX: CALCULATION OF TRANSITION RATES}

The transition rate is the conditional probability of jumping to a particular adjoining box per unit time. Thus, one possible way of deriving the transition rates is to first calculate the expected first exit time of a particle in a domain and then condition it based on the probability of either a left or right transition.

Although the first exit time will have a distribution, we appeal to the central limit theorem [36] and assume that we are using sufficiently large populations of particles to justify the claim that the first exit time will be close to the mean and have small variance. This assumption also needs to be fulfilled in order to use the linear noise expansion in Sec. II, where we need $\Omega$ to be large enough to justify the separation of various orders.

Although other approaches are possible [15,37,38], we use Dynkin's formula applied to the case of diffusion [39] to derive the exit time. The expected first exit time $\langle\tau\rangle$ of a random walk with diffusion coefficient $D$, from the interval $[-R, R]$ starting at a point $a \in[-R, R]$ is given by

$$
2 D\langle\tau\rangle=R^{2}-a^{2} .
$$

Hence, for the case of MMD, we start at $\left(x_{i}+x_{i-1}\right) / 2$, and we want to know when we first leave the region $\left[\left(x_{i-2}+\right.\right.$ $\left.\left.x_{i-1}\right) / 2,\left(x_{i}+x_{i+1}\right) / 2\right]$. Translating these points to be around zero gives

$$
\begin{gathered}
R=\frac{1}{2}\left(\frac{x_{i}+x_{i+1}}{2}-\frac{x_{i-2}+x_{i-1}}{2}\right), \\
=\frac{x_{i}+x_{i+1}-x_{i-2}-x_{i-1}}{4}, \\
a=\frac{x_{i}+x_{i-1}}{2}-\frac{1}{2}\left(\frac{x_{i}+x_{i+1}}{2}+\frac{x_{i-2}+x_{i-1}}{2}\right), \\
=\frac{x_{i}+x_{i-1}-x_{i-2}-x_{i+1}}{4} .
\end{gathered}
$$

Thus,

$$
2 D\langle\tau\rangle=\frac{\left(x_{i+1}-x_{i-1}\right)\left(x_{i}-x_{i-2}\right)}{4} .
$$

Hence, $1 /\langle\tau\rangle$ is the expected rate of leaving the interval. However, this rate has not been conditioned on to which adjoining Lagrangian box the particle diffuses.

By considering a simple random walk process the probability of hitting the left-hand boundary first, when starting at $a$, is [36]

$$
p_{l}=\frac{R-a}{2 R}=\frac{x_{i+1}-x_{i-1}}{x_{i}+x_{i+1}-x_{i-2}-x_{i-1}} .
$$


Similarly, the probability of hitting the right-hand boundary first is

$$
p_{r}=\frac{R+a}{2 R}=\frac{x_{i}-x_{i-2}}{x_{i}+x_{i+1}-x_{i-2}-x_{i-1}} .
$$

Combining these probabilities with the transition rates gives the conditional transition rates of jumping left $l_{i}$ and jumping right $r_{i}$ from the midpoint of a Lagrangian box to the midpoint of the adjoining Lagrangian box:

$$
\begin{aligned}
l_{i} & =\frac{p_{l}}{\langle\tau\rangle}=\frac{8 D}{\left(x_{i}-x_{i-2}\right)\left(x_{i}+x_{i+1}-x_{i-2}-x_{i-1}\right)}, \\
r_{i} & =\frac{p_{r}}{\langle\tau\rangle}=\frac{8 D}{\left(x_{i+1}-x_{i-1}\right)\left(x_{i}+x_{i+1}-x_{i-2}-x_{i-1}\right)} .
\end{aligned}
$$

The case of AMMD is treated similarly:

$$
\begin{aligned}
R & =x_{i}-x_{i+1}, \\
a & =0, \\
2 D\langle\tau\rangle & =\left(x_{i}-x_{i+1}\right)^{2}, \\
p_{l} & =\frac{1}{2},
\end{aligned}
$$

$$
p_{r}=\frac{1}{2} \text {. }
$$

We then extend these descriptions to the case of a stochastically growing domain and use $\left(x_{i}-x_{i-1}\right)=\Delta_{E} N_{i}$. Remembering that $\theta=\Delta_{L} / \Delta_{E}$ and $D=d_{L} \Delta_{L}^{2}$ we can derive the following expressions for the dependency of stochastic diffusion on growth:

MMD transition rates from Lagrangian box $i$ :

$$
\begin{aligned}
& l_{i}=\frac{8 d_{L}}{\frac{1}{\theta^{2}}\left(N_{i}+N_{i-1}\right)\left(N_{i-1}+2 N_{i}+N_{i+1}\right)}, \\
& r_{i}=\frac{8 d_{L}}{\frac{1}{\theta^{2}}\left(N_{i}+N_{i+1}\right)\left(N_{i-1}+2 N_{i}+N_{i+1}\right)} .
\end{aligned}
$$

AMMD transition rates from Lagrangian box $i$ :

$$
\begin{aligned}
& l_{i}=\frac{d_{L}}{\frac{1}{\theta^{2}} N_{i}^{2}}, \\
& r_{i}=\frac{d_{L}}{\frac{1}{\theta^{2}} N_{i}^{2}} .
\end{aligned}
$$

[1] B. Alberts, D. Bray, J. Lewis, M. Raff, K. Roberts, and J. D. Watson, Molecular Biology of the Cell, 3rd ed. (Garland Science, New York, 1994).

[2] J. D. Murray, Mathematical Biology II: Spatial Models and Biomedical Applications, 3rd ed., Vol. 2 (Springer, Berlin, 2003).

[3] A. M. Turing, Philos. Trans. R. Soc. London B 237, 37 (1952).

[4] J. Bard and I. Lauder, J. Theor. Biol. 45, 501 (1974).

[5] B. Bunow, J. P. Kernevez, G. Joly, and D. Thomas, J. Theor. Biol. 84, 629 (1980).

[6] R. E. Baker, E. A. Gaffney, and P. K. Maini, Nonlinearity 21, R251 (2008).

[7] R. Erban, S. J. Chapman, and P. K. Maini, e-print arXiv:0704.1908v2 [q-bio.SC].

[8] E. J. Crampin, E. A. Gaffney, and P. K. Maini, Bull. Math. Biol. 61, 1093 (1999).

[9] P. M. Kulesa, G. C. Cruywagen, S. R. Lubkin, M. W. J. Ferguson, and J. D. Murray, Acta Biotheor. 44, 153 (1996).

[10] P. M. Kulesa, G. C. Cruywagen, S. R. Lubkin, P. K. Maini, J. Sneyd, M. W. J. Ferguson, and J. D. Murray, J. Theor. Biol. 180, 287 (1996).

[11] S. Kondo and R. Asai, Nature (London) 376, 765 (1995).

[12] S. A. Newman, S. Christley, T. Glimm, H. G. E. Hentschel, B. Kazmierczak, Y. T. Zhang, J. Zhu, and M. Alber, Curr. Top. Dev. Biol. 81, 311 (2008).

[13] R. E. Baker, C. A. Yates, and R. Erban, Bull. Math. Biol. 72, 719 (2010).

[14] T. E. Woolley, R. E. Baker, E. A. Gaffney, and P. K. Maini, Phys. Rev. E 84, 021915 (2011).

[15] N. G. van Kampen, Stochastic Processes in Physics and Chemistry, 3rd ed. (North Holland, Amsterdam, 2007).

[16] E. J. Hinch, Perturbation Methods (Cambridge University Press, Cambridge, 1991).

[17] J. D. Murray, Mathematical Biology I: An Introduction, 3rd ed., Vol. 1 (Springer, Berlin, 2003).

[18] J. D. Murray, E. A. Stanley, and D. L. Brown, Proc. R. Soc. London B 229, 111 (1986).
[19] A. Okubo, P. K. Maini, M. H. Williamson, and J. D. Murray, Proc. R. Soc. London B 238, 113, (1989).

[20] T. E. Woolley, R. E. Baker, E. A. Gaffney, and P. K. Maini, Mathematical Modelling of Zombies (to be published).

[21] D. T. Gillespie, Annu. Rev. Phys. Chem. 58, 35 (2007).

[22] H. Risken, The Fokker-Planck Equation: Methods of Solution and Applications (Springer, Berlin, 1989).

[23] J. Elf and M. Ehrenberg, Genome. Res. 13, 2475 (2003).

[24] S. S. Andrews and D. Bray, Phys. Biol. 1, 137 (2004).

[25] D. A. McQuarrie, J. Appl. Prob. 4, 413 (1967).

[26] C. W. Gardiner, K. J. McNeil, D. F. Walls, and I. S. Matheson, J. Stat. Phys. 14, 307 (1976).

[27] K. W. Morton and D. F. Mayers, Numerical Solution of Partial Differential Equations: An Introduction (Cambridge University Press, Cambridge, 2005).

[28] A. J. McKane and T. J. Newman, Phys. Rev. Lett. 94, 218102 (2005).

[29] A. J. McKane, J. D. Nagy, T. J. Newman, and M. O. Stefanini, J. Stat. Phys. 128, 165 (2007).

[30] C. A. Lugo and A. J. McKane, Phys. Rev. E 78, 51911 (2008).

[31] T. Butler and N. Goldenfeld, Phys. Rev. E 80, 30902 (2009).

[32] W. L. Briggs and V. E. Henson, The DFT: An Owner's Manual for the Discrete Fourier Transform (SIAM, Philadelphia, 1995).

[33] T. Biancalani, D. Fanelli, and F. Di Patti, Phys. Rev. E 81, 046215 (2010).

[34] J. D. Murray, J. Theor. Biol. 98, 143 (1982).

[35] T. E. Woolley, R. E. Baker, E. A. Gaffney, and P. K. Maini, Phys. Rev. E 84, 021915 (2011).

[36] G. Grimmett and D. Stirzaker, Probability and Random Processes (Oxford University Press, New York, 2001).

[37] S. Redner, A Guide to First-Passage Processes (Cambridge University Press, Cambridge, 2001).

[38] K. K. J. Kinateder, Stat. Prob. Lett. 51, 1 (2001).

[39] B. K. Øksendal, Stochastic Differential Equations: An Introduction with Applications (Springer, Berlin, 2003). 